\title{
Weight and body composition in a cohort of HIV-positive men and women
}

\author{
JE Forrester ${ }^{1, *}$, D Spiegelman ${ }^{3}$, M Woods $^{1}$, TA Knox ${ }^{2}$, JM Fauntleroy $^{1}$ and SL Gorbach ${ }^{1,2}$ \\ 'Department of Family Medicine and Community Health, Tufts University School of Medicine, 136 Harrison Avenue \\ (Stearns 203A), Boston, MA 02111 , USA: '2Department of Medicine, New England Medical Center, Boston, \\ MA 02 1 11, USA: ${ }^{3}$ Departments of Epidemiology and Biostatistics, Harvard School of Public Health, Boston, \\ MA 02 115, USA
}

\begin{abstract}
Objective: At issue is whether weight loss in HIV infection is a cachectic process, characterised by loss of lean body mass with conservation of fat, or a process of starvation. We present data on body composition from 516 persons at different stages of HIV infection as determined by CD4 counts.

Design: Cross-sectional analyses of body composition in relation to CD4 count.

Setting: The baseline data from a prospective cohort study of outcomes in HIV/AIDS in relation to nutritional status in Boston, Massachusetts, USA.

Subjects: The first 516 subjects with HIV/AIDS to enrol in the study.

Results: Differences in weight in relation to CD4 counts were present only at CD4 counts of 600 or less (slope below CD4 $=600: 1.9 \mathrm{~kg}$ per 100 CD4 cells, $P<0.0001$ ). On average, $68 \%$ of the difference in weight over CD4 counts was fat (slope: $1.3 \mathrm{~kg}$ fat per 100 CD4 cells, $P<0.0001)$.

Conclusions: This cross-sectional analysis suggests that weight loss consists principally of fat loss in those persons with adequate fat stores. This observation will need to be confirmed in longitudinal analyses.
\end{abstract}

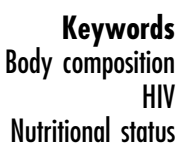

HIV wasting, or an unintentional weight loss of more than $10 \%$ of normal weight, is a common AIDS-defining condition $^{1}$. HIV-associated weight loss is an independent predictor of opportunistic infections, hospitalisation and mortality $^{2-7}$. Loss of lean body mass, however, is a better predictor of survival than weight loss $s^{2,5}$. The importance of lean body mass to survival in HIV has led to an interest in the changes in body composition that accompany disease progression, and the ability of appetite stimulants, anabolic steroids and human growth hormone to restore lean body mass ${ }^{8-11}$.

Early in the AIDS epidemic, Kotler and colleagues ${ }^{12}$ found that homosexual men with advanced AIDS had less body cell mass (the intracellular component of lean body mass), but similar amounts of body fat, compared with healthy homosexual men. It was postulated that HIV wasting is a cachectic process in which weight loss is disproportionately loss of lean body mass, with relative conservation of fat ${ }^{12-14}$. Loss of fat was deemed a poor marker of AIDS wasting ${ }^{12,13}$. Proposed mechanisms for muscle wasting include altered cytokine levels ${ }^{13-16}$ and alterations in circulating levels of anabolic sex hormones ${ }^{17}$.
While some subsequent studies have, indeed, found that weight loss is mainly loss of lean body mass ${ }^{18,19}$, other studies have found principally fat loss or a loss of both fat and lean ${ }^{20-23}$. A loss of lean body mass with conservation of fat suggests a cachectic process; a loss of mainly fat would be consistent with wasting due to malnutrition. The discrepancies among these studies may be due to differences in the stage of disease and, possibly, differences in initial body composition. Studies of healthy adults who have lost weight due to caloric restriction have shown that loss of lean body mass is a function of body fat ${ }^{24-26}$. Mulligan and colleagues ${ }^{23}$, based on studies of 32 men, proposed that loss of lean body mass in persons with HIV, like healthy adults, is dependent on the percentage of body fat prior to the weight loss.

Here, we report findings from a cross-sectional analysis of weight and body composition in relation to CD4 cell counts from the baseline data of a three-year longitudinal, observational study of a cohort of more than 500 ambulatory persons with HIV. This cohort includes 130 women, a group that has been under-represented in previous studies. 


\section{Methods}

\section{Participants}

The Tufts University Nutrition for Healthy Living Study, initiated in February of 1995, aims to describe the natural history of HIV wasting and to determine the role of nutrition in the progression of HIV disease. Recruitment of participants was done through advertisements on the radio, in local newspapers, health clinics and physician networks. Persons 18 years of age and older who were HIV-positive and consented to participate were considered eligible. People who met any one of the following criteria were excluded: pregnant at recruitment, diagnosed diabetes mellitus, thyroid disease, or malignancies (other than HIV-associated malignancies), inadequate English language fluency. Clinic visits occurred at three sites: the New England Medical Center and the Fenway Community Health Center in Boston, and the Miriam Hospital in Providence, Rhode Island.

\section{Assessments}

The baseline measurements were made during two clinic visits approximately 10 days apart. Those persons who completed the full two-visit baseline protocol were considered to have enrolled in the study. Height $( \pm 0.1 \mathrm{~cm})$ and weight $( \pm 0.1 \mathrm{~kg})$ were measured at the first visit using standard techniques ${ }^{27}$. Body composition by bioelectrical impedance analysis (BIA) was determined at the second visit, after an overnight fast, following the manufacturer's recommended procedures (RJL Systems Inc., Clinton, MI) and using the equations of Lukaski ${ }^{28}$ to derive measures of fat-free mass (principally lean body mass and bone). Body mass index (BMI) was calculated by: WT $(\mathrm{kg}) / \mathrm{HT}^{2}\left(\mathrm{~m}^{2}\right)$. CD lymphocyte subset counts were done by standard techniques at clinical laboratories of the participating hospitals. Confidentiality of records was preserved according to regulations stipulated by human studies review committees at each participating institution. The protocol was approved by institutional review boards at each site.

\section{Statistical analyses}

The data were double entered and checked using MS Access (Microsoft Corp., Seattle, WA). All analyses were done in the generalised linear models program of SAS version 6.1 (SAS Institute, Cary, NC). We compared weight and body composition separately for men and women, categorised by level of CD4 count. Differences in weight, BMI, fat mass and fat-free mass across the CD4 count categories were tested for significant trends by assigning each subject to one of three CD 4 categories: less than 200, 200 to 499 , or greater than or equal to 500. A score of one, two or three was assigned to the CD4 categories, respectively, and each of the four outcomes (weight, $\mathrm{BMI}$, fat mass and fat-free mass) was regressed on the score.
In order to examine the shape of the relationship between weight, body composition and CD4 counts, weight, fat and fat-free mass were regressed on CD4 counts treated as a continuous variable. To account for possible non-linear relationships, linearity was assessed using the technique of restricted cubic splines with three-, four- and five-knot models ${ }^{29}$. When the hypothesis of linearity was rejected at $P<0.1$, a non-linear regression model was fit by least squares to a change-point model to locate the point where the slope of the line changed. Residuals of all models were checked for violations of assumptions. Influential points were identified by Cook's distance. Variables describing the use (yes/no) of protease inhibitors, non-nucleoside reverse transcriptase inhibitors and anabolic steroids were included in the models. The medication variables were used as interaction terms (with CD4 counts) to assess whether the slopes observed differed between persons taking these medications and persons not taking such medications. Nucleotide and nucleoside reverse transcriptase inhibitors were not evaluated, as the majority (76\%) of the study subjects was taking them. Separate analyses were performed for men and women. The data for men and women were combined if analyses showed no significant betweengender difference in the estimated slopes. Alpha (twotailed) was set at 0.05 unless otherwise stated.

Data on body composition were missing for six men. CD4 counts were unavailable for three women because the phlebotomist was unable to draw blood. Two of these women reported their CD4 counts to be less than 200; their CD4 counts were set to a value of 100 (midpoint of 0 and 200). CD4 counts from the six-month protocol visit (six-month visit CD4 count $=541$ ) were used for the third woman.

\section{Results}

As of December 1997, 516 persons were enrolled in the study. Three-hundred-and-eighty-six (75\%) of the participants were men and 130 (25\%) were women. The racial composition of the men was as follows: 276 (71\%) Caucasian, 72 (19\%) African-American, 23 (6\%) Hispanic, 15 (4\%) other races. Fifty-seven (44\%) of the women were Caucasian, 50 (38\%) African-American, 15 (12\%) Hispanic and eight $(6 \%)$ were of other races. One-hundred-andsixty-three (42\%) of the men in the cohort and 87 (67\%) of the women reported incomes $\leq \$ 10000$ per year.

Forty-nine per cent (193/394) of the subjects were taking one of a protease inhibitor class, non-nucleoside reverse transcriptase inhibitor class or an anabolic steroid class of medications. Information on these medications was missing for 122 of the subjects, in most cases (76\%) because the participant was concurrently enrolled in a blinded clinical trial.

The mean weight, body mass index (BMI) and body composition of the men and women in relation to CD4 
Table 1 Age, body weight and body composition in relation to CD4 class (mean \pm SD)

\begin{tabular}{|c|c|c|c|c|c|}
\hline \multirow[b]{2}{*}{ Characteristic } & & \multicolumn{3}{|c|}{ CD4 counts } & \multirow[b]{2}{*}{$P$ for trend } \\
\hline & & $<200$ & $200-499$ & $\geq 500$ & \\
\hline \multirow{2}{*}{ Number in subgroup } & $\mathrm{M}$ & 136 & 171 & 79 & \\
\hline & $\mathrm{F}$ & 30 & 63 & 37 & \\
\hline \multirow{2}{*}{ Age (years) } & $\mathrm{M}$ & $40(7.3)$ & $41(7.6)$ & 38 (7.9) & \\
\hline & $\mathrm{F}$ & 39 (7.9) & $39(7.7)$ & $37(6.5)$ & \\
\hline \multirow[t]{2}{*}{ Weight $(\mathrm{kg})$} & $\mathrm{M}$ & 72.1 (11.5) & $76.8(11.1)$ & 80.5 (12.6) & 0.0001 \\
\hline & $\mathrm{F}$ & $62.8(13.5)$ & $69.3(16.7)$ & $72.8(15.7)$ & 0.0090 \\
\hline \multirow[t]{2}{*}{ Body mass index $\left(\mathrm{kg} \mathrm{m}^{-2}\right)$} & M & $23.2(3.1)$ & $24.9(3.1)$ & $25.8(3.8)$ & 0.0001 \\
\hline & $\mathrm{F}$ & $23.8(4.6)$ & $26.9(6.6)$ & $27.2(6.0)$ & 0.025 \\
\hline \multirow[t]{2}{*}{ Body fat (kg) } & M & $13.4(6.8)$ & $16.8(7.5)$ & $19.8(9.4)$ & 0.0001 \\
\hline & $\mathrm{F}$ & $20.5(9.1)$ & $25.6(12.7)$ & 26.2 (11.5) & 0.045 \\
\hline \multirow[t]{2}{*}{ Fat-free mass $(\mathrm{kg})$} & $\mathrm{M}$ & 58.5 (7.3) & $60.2(7.2)$ & $60.7(6.4)$ & 0.030 \\
\hline & $\mathrm{F}$ & $42.4(6.7)$ & $43.8(6.2)$ & $46.6(6.2)$ & 0.0066 \\
\hline \multirow[t]{2}{*}{$\%$ Body fat } & $\mathrm{M}$ & $18.0(6.9)$ & $21.3(7.1)$ & $23.7(8.0)$ & 0.0001 \\
\hline & $\mathrm{F}$ & $31.5(8.3)$ & $35.2(9.6)$ & $34.6(8.3)$ & 0.15 \\
\hline \multirow{2}{*}{$\%$ Lean body mass } & $\mathrm{M}$ & $82.0(6.7)$ & $78.7(7.1)$ & $76.2(8.0)$ & 0.0001 \\
\hline & $\mathrm{F}$ & $68.5(8.3)$ & $64.8(9.6)$ & $65.4(8.3)$ & 0.15 \\
\hline
\end{tabular}

count categories are shown in Table 1. There was a significant trend towards lower weight and BMI with lower CD 4 counts. The differences in weight and BMI among the CD4 groups were due to both lower body fat and lower fat-free mass at lower CD4 counts, although the difference in body fat was greater than the difference in fat-free mass, as reflected by the higher per cent fat-free mass in the CD 4 count category $<200$ compared with the higher CD4 count categories.

When we treated CD 4 counts as a continuous variable to examine the shape of the relationship between weight, body composition and CD 4 counts, we found that, in the men, the cross-sectional relation between CD 4 counts and the outcome was non-linear for weight $(P=0.028)$ and fat-free mass $(P=0.030)$, and of borderline significance for body fat $(P=0.13)$ (Figs $1-3)$. In the women, the hypothesis of non-linearity did not reach statistical significance, implying that the relationships between CD4 counts and weight, fat mass and fat-free mass were

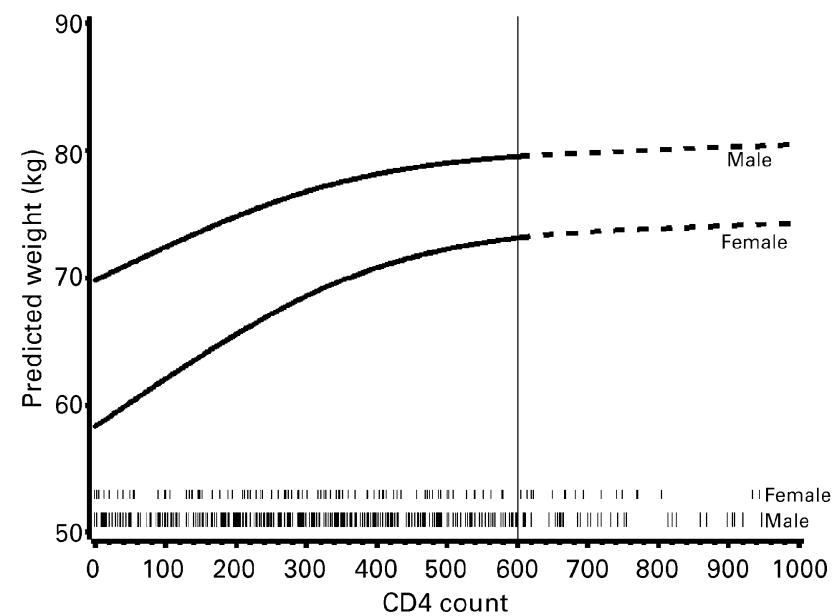

Fig. 1 The relation of weight $(\mathrm{kg})$ to CD4 counts. The dotted line indicates a slope not significantly different from zero linear in the women (Figs 1-3). However, since the curves for men and women were of similar shape, we treated the relations between CD 4 counts and weight, fat and fat-free mass as non-linear for women in subsequent analyses.

In the subgroup with CD4 cell counts above 600, there was no significant difference in weight among subjects at different levels of CD 4 counts (slope: $0.30 \mathrm{~kg}$ per $100 \mathrm{CD} 4$ counts, $\mathrm{SE}=0.73, \quad P=0.68$ ). Among those persons whose CD4 counts were below 600, average weight was less with lower CD 4 cell counts. Weight was, on average, $1.9 \mathrm{~kg}$ lower for each decline of $100 \mathrm{CD} 4$ cells $(P<$ 0.0001). There was no difference between men and women in the slope describing the relationship between weight and CD 4 counts (difference in slopes: $-0.85 \mathrm{~kg}$ per $100 \mathrm{CD} 4$ counts, $\mathrm{SE}=0.85, P=0.32$ ) in the subgroup with CD 4 counts below 600 , so the estimate of $1.9 \mathrm{~kg}$ per 100 CD4 cell difference comes from a model that combined the data from both men and women. Among those persons whose CD 4 counts were below 600, the

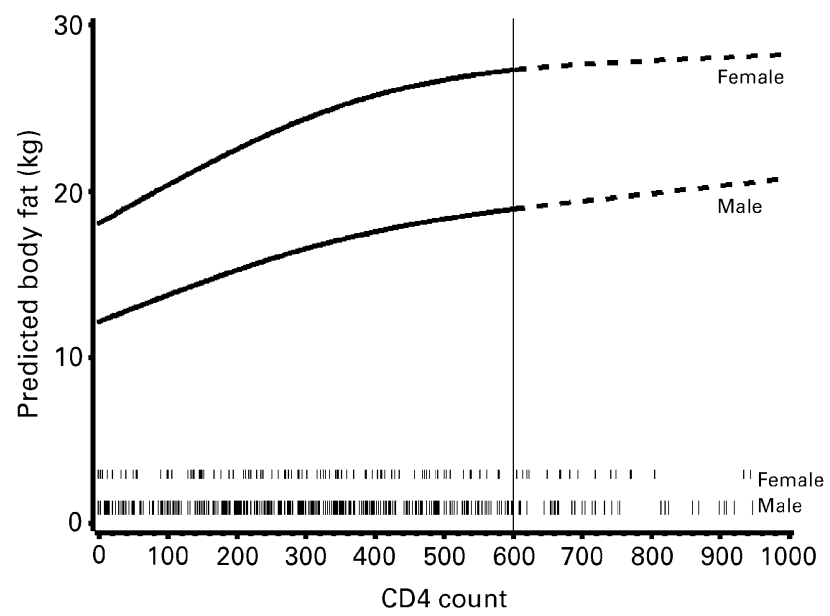

Fig. 2 The relation of fat $(\mathrm{kg})$ to CD4 counts. The dotted line indicates a slope not significantly different from zero 


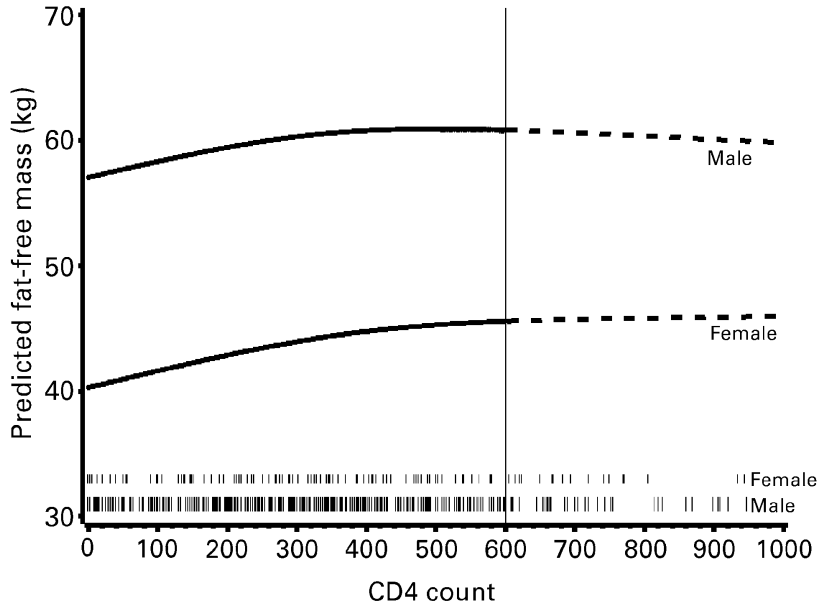

Fig. 3 The relation of fat-free mass $(\mathrm{kg})$ to CD4 counts. The dotted line indicates a slope not significantly different from zero

average difference in fat was $1.3 \mathrm{~kg}(P<0.0001)$ and difference in fat-free mass was $0.65 \mathrm{~kg}(P=0.011)$ per difference of 100 in CD4 cells. Similar to the results for weight, there was no difference between men and women in the slope of the line describing the crosssectional relationship between CD4 counts and kilograms of fat or fat-free mass (between-sex difference in slopes for fat: $0.84 \mathrm{~kg}$ per $100 \mathrm{CD} 4$ counts, $\mathrm{SE}=0.58, P=0.15$; for fat-free mass: $-0.10 \mathrm{~kg}$ per $100 \mathrm{CD} 4$ counts, $\mathrm{SE}=$ $0.48, P=0.83)$. Thus, fat accounted for an average of $68 \%$ (1.3 kg fat $/ 1.9 \mathrm{~kg}$ weight) of the difference in weight across CD 4 counts in those persons with CD4 values below 600 . The addition of interaction terms showed no evidence that the estimated slopes differed between those persons on protease inhibitors, non-nucleoside reverse transcriptase inhibitors and/or anabolic steroids and those who were not taking medications of this type ( $P$ for the difference in slopes: 0.62).

\section{Discussion}

Differences in weight and body composition were observed in subjects with CD4 counts less than 600 . These data support the notion that weight loss starts early in HIV infection while CD4 counts are still above the range associated with opportunistic infections. Our crosssectional data also suggest that weight loss in HIV infection is mostly fat loss in those persons with adequate fat stores. Our data do not support the theory that HIV wasting is principally a loss of lean body mass with fat conservation, believed to be typical of a cachectic process.

Some previous studies have found HIV-associated weight loss to consist of greater loss of lean body mass than fat mass ${ }^{12-14,18,19}$. Other studies have shown a greater loss of fat than lean body mass or loss of both fat and lean ${ }^{20-23}$. The apparent contradictions may be explained, in part, by differences in the average per cent body fat of the study subjects. Mulligan and colleagues ${ }^{23}$, in a longitudinal analysis of weight loss in 32 men with HIV, found that both fat and lean were lost and that the composition of the weight loss depended on the baseline body fat composition, such that the men with initially higher per cent body fat lost greater amounts of fat than lean body mass. The high average per cent body fat in the men and women in our study within all CD4 count classes would explain the predominance of a cross-sectional difference in fat vs. lean. Our data also suggest that the pattern of weight differences across CD4 counts is similar in men and women.

The differences observed in body composition across CD4 classes in these people with HIV appear to be qualitatively similar to the changes expected in healthy people who have lost weight due to caloric restriction. Forbes $^{25}$, summarising data from several independent studies, hypothesised that in healthy persons losing weight due to caloric restriction, the proportion of weight loss due to loss of lean body mass is inversely related to body fat. Dulloo and colleagues ${ }^{26}$ found that, in healthy men undergoing semi-starvation, weight loss was principally fat in those with adequate fat stores.

It is, perhaps, not surprising that our estimates of the cross-sectional relation between weight, body composition and CD4 counts did not differ between those persons on protease inhibitor therapies and/or anabolic steroids and those not taking these medications. Protease inhibitor therapy does not inevitably increase CD4 counts, nor does it have a large effect on weight ${ }^{30}$. Clinical trials of treatments for wasting using appetite stimulants, anabolic steroids and growth hormone have shown only modest increases in weight and lean body mass ${ }^{8-11}$.

Our participants were all healthy enough to participate in a rigorous protocol, and so these results cannot be generalised to HIV patients who were too ill to participate in our study. There may be important differences in the changes in body composition that occur as the disease advances, between persons who have AIDS or are suffering from active opportunistic conditions and those who are not. These findings may also be of limited generalisability, quantitatively, to persons with untreated HIV infection or to persons with pre-existing malnutrition, as would be common in developing countries. Qualitatively, however, the evidence of weight differences between CD 4 groups at CD 4 counts well above 200, and the predominance of differences in fat (vs. fat-free mass), should be generalisable to persons who have similar fat stores and who are in the early stages of HIV infection, whether the infection is treated or untreated.

More data from large-scale longitudinal studies are needed to examine the changes in body composition that accompany HIV disease progression, and to determine if the maintenance of adequate fat stores early in HIV infection will help prevent the loss of lean body mass and 
improve long-term survival. In countries where the use of protease inhibitors for the treatment of HIV infection is widespread, the reported changes in body composition and fat distribution associated with therapy may complicate such studies. Nevertheless, studies of this type are feasible, are of relatively low cost, and the results potentially important for countries where expensive medications are not available.

\section{Contributions}

JEF contributed to the analyses, interpretation and writing of the manuscript. DS contributed to the study design, analyses and interpretation of the results. MW contributed to the study design and interpretation of the results. TK contributed to the study design and coordination of the data collection. JMF contributed to the analyses and interpretation of the results. SLG contributed to the study design and oversaw the project.

\section{Acknowledgements}

This study was supported by NIDDK grants DK4 5734-03 and NIDA grant DA 11598. The General Clinical Research Center of the New England Medical Center, Boston, MA is supported by the Division of Research Resources of the NIH, grant number MO1-RR00054. We wish to thank the participants of the study for their time and enthusiasm. Thanks to the CSU team for their diligence.

\section{References}

1 CDC, US Department of Health and Human Services. HIV/ AIDS Surveillance Report 1998; 9(2).

2 Kotler DP, Tierney AR, Wang J, et al. Magnitude of body cell mass depletion and timing of death from wasting in AIDS. Am.J. Clin. Nutr. 1989; 50: 444-7.

3 Chelbowski RT, Grosvenor MB, Bernhard NH, et al. Nutritional status, gastrointestinal dysfunction, and survival in patients with AIDS. Am. J. Gastroenterol. 1989; 84: 1288-93.

4 Guenter P, Muurahainen N, Simons G, et al. Relationships among nutritional status, disease progression, and survival in HIV infection. J. Acquired Immune Defic. Syndr. 1993; 6: 1130-8.

5 Suttmann U, Ockenga J, Selberg O, et al. Incidence and prognostic value of malnutrition and wasting in human immunodefiency virus-infected outpatients. J. Acquired Immune Defic. Syndr. 1995; 8: 239-46.

6 Palenicek JG, Graham NMH, He YH, et al. Weight loss prior to clinical AIDS as a predictor of survival. J. Acquired Immune Defic. Syndr. 1995; 10: 366.

7 Wheeler DA, Gilbert CL, Launer CA, et al. Weight loss as a predictor of survival and disease progression in HIV infection. J. Acquired Immune Defic. Syndr. 1998; 18: 80-5.

8 Engelson ES, Pi-Sunyer FX, Kotler DP. Effects of megesterol acetate therapy on body composition and circulating testosterone levels in patients with AIDS. AIDS 1995; 9: $1107-8$.

9 Gold J, High HA, Li Y, et al. Safety and efficacy of nandrolone decanoate for treatment of wasting in patients with HIV infection. AIDS 1996; 10: 745-52.
10 Schambelan M, Mulligan K, Grunfels C, et al. Recombinant human growth hormone in patients with HIV-associated wasting. Ann. Intern. Med. 1996; 125: 873-82.

11 Waters D, Danska J, Hardy K, et al. Recombinant human growth hormone, insulin-like growth factor 1 , and combination therapy in AIDS-associated wasting. Ann. Intern. Med. 1996; 125: 865-72.

12 Kotler DP, Wang J, Pierson RN. Body composition studies in patients with the acquired immunodeficiency syndrome. Am. J Clin. Nutr. 1985; 42: 1255-65.

13 Grunfeld C, Feingold KR. Metabolic disturbances and wasting in the acquired immunodeficiency syndrome. $N$. Engl. J. Med. 1992; 327: 329-37.

14 Babameto G, Kotler DP. Malnutrition in HIV infection. Gastroenterol. Clin. North Am. 1997; 26: 393-415.

15 Hellerstein MK, Grunfeld C, Wu K, et al. Increased de novo hepatic lipogenesis in human immunodeficiency virusinfected humans. J. Clin. Endocrinol. Metab. 1993; 76: 559-65.

16 Mitch WE, Goldberg AL. Mechanisms of muscle wasting. $N$. Engl. J. Med. 1996; 335: 1897-905.

17 Grinspoon S, Corcoran C, Lee K, et al. Loss of lean body and muscle mass correlates with androgen levels in hypogonadal men with acquired immunodeficiency syndrome and wasting. J. Clin. Endocrinol. Metab. 1996; 81: 4051-8.

18 Ott M, Lambke B, Fischer H, et al. Early changes of body composition in human immunodeficiency virus-infected patients: tetrapolar body impedance analysis indicates significant malnutrition. Am. J. Clin. Nutr. 1993; 57: 15-9.

19 Paton NIJ, Macallan DC, Jebb SA, et al. Longitudinal changes in body composition measured with a variety of methods in patients with AIDS. J. Acquired Immune Defic. Syndr. 1997; 14: 119-27.

20 Schwenk A, Hoffer-Belitz E, Jung B, et al. Resting energy expenditure, weight loss and altered body composition in HIV infection. Nutrition 1996; 12: 595-601.

21 Sharkey SJ, Sharkey KA, Sutherland LR, et al. Nutritional status and food intake in human immunodeficiency virus infection. J. Acquired Immune Defic. Syndr. 1992; 5: 1091-8.

22 Grinspoon S, Corcoran C, Miller K, et al. Body composition and endocrine function in women with aquired immunodeficiency syndrome wasting. J. Clin. Endocrinol. Metab. 1997; 82: $1332-7$.

23 Mulligan K, Tai VW, Schamblean M. Cross-sectional and longitudinal evaluation of body composition in men with HIV. J. Acquired Immune Defic. Syndr. 1997; 15: 43-8.

24 Durrant ML, Garrow JS, Royston P, et al. Factors influencing the composition of the weight lost by obese patients on a reducing diet. Br. J. Nutr. 1980; 44: 275-85.

25 Forbes GB. Lean body mass--body fat interrelationships in humans. Nutr. Rev. 1987; 45: 225-31.

26 Dulloo AG, Jacquet J, Girardier L. Autoregulation of body composition during weight recovery in humans: the Minnesota experiment revisited. Int. J. Obes. Relat. Metab. Disord. 1996; 20: 393-405.

27 Lohman TG, Roche AF, Martorell R, eds. Anthropometric Standardization Reference Manual. Champaign, IL: Human Kinetics Books, 1988.

28 Lukaski HC. Use of bioelectrical impedance analysis to assess human body composition: a review. In: Livingston GE, ed. Nutritional Status Assessment of the Individual. Trunbull, CT: Food \& Nutrition Press, Inc., 1989; 189-204.

29 Durrleman S, Simon R. Flexible regression models with cubic splines. Stat. Med. 1989; 8: 551-61.

30 Silva M, Skolnick PR, Gorbach SL, et al. The effect of protease inhibitors on weight and body composition in HIV-infected patients. AIDS 1998; 12: 1645-51. 